Research Article

\title{
Analysis of the Influence of Inlet Guide Vanes on the Performance of Shaft Tubular Pumps
}

\author{
Haifeng Jiao $\mathbb{D}^{1},{ }^{1}$ Chong Sun $(\mathbb{D})^{2}$ and Songshan Chen ${ }^{3}{ }^{3}$ \\ ${ }^{1}$ College of Hydraulic Science and Engineering, Yangzhou University, Yangzhou 225000, Jiangsu, China \\ ${ }^{2}$ Shandong Water Diversion Project Operation and Maintenance Center, Jinan 250000, Shandong, China \\ ${ }^{3}$ College of Electrical, Energy and Power Engineering, Yangzhou University, Yangzhou 225000, Jiangsu, China
}

Correspondence should be addressed to Chong Sun; yzcss08@163.com

Received 7 June 2021; Accepted 28 October 2021; Published 16 December 2021

Academic Editor: M.I. Herreros

Copyright (C) 2021 Haifeng Jiao et al. This is an open access article distributed under the Creative Commons Attribution License, which permits unrestricted use, distribution, and reproduction in any medium, provided the original work is properly cited.

To study the influence of inlet guide vanes (IGVs) on the pressure pulsation of a shaft tubular pump, this paper first conducts an experiment to study IGVs. Then, numerical calculations of the shaft tubular pump with and without IGVs are performed to analyze the hydraulic performance and pressure fluctuation characteristics. Finally, the reliability and accuracy of the data are verified by a model test. Numerical simulation results show that with additional IGVs, the pressure pulsation amplitude at the impeller inlet first decreases and then increases under small-flow and design conditions but gradually increases under large-flow conditions. When the IGVs are added to the impeller inlet of the shaft tubular pump, the hydraulic loss in front of the impeller inlet increases, resulting in a significant drop in the head and efficiency of the pump device when the flow rate is less than $1.12 Q_{\mathrm{d}}$; when the flow rate is greater than $1.12 Q_{\mathrm{d}}$, the head and efficiency of the pump device do not change significantly. IGVs can improve the condition of impeller water inflow and reduce pressure fluctuation on the blade surface.

\section{Introduction}

A shaft tubular pump is a kind of axial flow pump. In China, shaft tubular pumps are widely used in large-flow and lowhead pump stations. The shaft tubular pump has the advantages of straight flow passage, smooth internal flow, and small hydraulic loss [1-5]. However, in the actual construction process of the shaft tubular pumping station, the size of the pump is large, and the flow channel usually adopts a reinforced concrete structure. The tensile performance is poor, so it is necessary to add IGVs at the impeller inlet to support the flow channel. The addition of IGVs can not only improve the overall structural strength of the inlet channel but also improve the flow pattern of the pump. Therefore, the study of IGVs has great reference significance for engineering practice. In addition to its application in water pumps, IGVs are also widely used in compressors, gas turbines, and other applications [6-12].

In the present paper, the airfoil design and placement angle of the IGVs are based on the research results of some experts on centrifugal pumps [13-15]. Wang [16] et al. studied the effect of IGVs on unsteady flow in centrifugal pumps. The results showed that after installing the IGVs of $-36^{\circ}$ to $36^{\circ}$, the pressure fluctuation in the impeller and volute changed a little. After installing IGVs of $-60^{\circ}$ and $+60^{\circ}$, the pressure pulsation amplitude increased significantly due to the blocking effect of the IGVs. Liu [17] et al. studied the difference in pressure pulsation in centrifugal pumps without IGVs and with 2D and 3D IGVs. The study found that under the design conditions, the main frequency amplitude of the pump was reduced by installing IGVs at the impeller inlet. 3D IGVs had a better reduction effect on the main frequency of pressure pulsation than 2D IGVs. Shi et al. [18] studied the effect of the angle difference of impeller blades on the performance of an axial flow pump. The results showed that the blade angle deviation caused irregular pressure changes in the impeller, and the low-frequency pressure pulsation increased significantly. Yang et al. [19] designed several IGVs of axial flow pumps with different installation angles. Through numerical calculation, it was 
found that IGVs with a $0^{\circ}$ angle had little effect on the hydraulic characteristics of the pumps in high-efficiency areas and low-flow conditions. The larger hydraulic loss from the IGVs was the main reason for the low efficiency of the pumps under large-flow conditions.

There have been a relatively large number of studies on rear guide vanes [20-26]. Yang et al. [27] studied the angle of the IGVs of an axial flow pump. When the angle of the blade was adjusted from positive to negative, the performance curve shifted in the direction of large flow. Zhao et al. [28] studied the IGVs of an axial flow pump in turbine mode. It was found that the efficiency range of the pump was expanded by the IGVs. Kim et al. [29] found that IGVs greatly interfered with the steady and unsteady characteristics of a pump. In particular, the efficiency was most affected by the IGVs. Guo et al. [30] found that the installation angle of IGVs had a greater impact on the cavitation of a pump. Yang et al. [31] studied the influence of the impeller inlet baffle on its performance. It was found that the distance between the inlet baffle and the impeller had the greatest impact on water pump performance. The inlet baffle could constrain the backflow of the impeller inlet and improve the efficiency of the pump. Xing et al. [32] set a baffle plate at the impeller inlet and analyzed it numerically. The study found that the baffle plate mainly affected the tangential velocity of the impeller inlet and could improve the impeller working ability. Dai et al. [33] researched the positions of IGVs. The closer the IGVs were to the impeller, the better the working condition of the impeller was. The efficiency of the pump set was also improved. Deng et al. [34] found that IGVs had a substantial impact on the external characteristics and vibration characteristics of pumps.

The present paper adopts a research method that combines numerical simulation and model tests [35-38]. First, the influence of the number of IGVs on the performance and pressure pulsation of the shaft tubular pump is studied through model tests. Then, the 5 IGVs scheme, which offers better performance than other schemes, and the shaft tubular pump without IGVs are selected for numerical simulation and comparison. Finally, several model tests are used to prove the accuracy of the calculation data. We mainly analyze the influence of the number of IGVs and the addition of IGVs on the performance and pressure pulsation characteristics of the pump unit. The purpose of this article is to derive the change law of the pressure pulsation of the shaft tubular pump [39-41] and provide guidance for the structural design and operation of pump stations.

\section{Model Test}

2.1. Test Scheme Design. A shaft tubular pump without IGVs and a shaft tubular pump with 4 IGVs, 5 IGVs, and 6 IGVs were designed. Tests on the external characteristics and pressure fluctuations were performed on a hydraulic machinery test bench that was accurately calibrated. The system error $e_{S}$ and measurement error $e_{R}$ of the test bench are $\pm 0.231 \%$ and $\pm 0.171 \%$. The test speed is the same as the calculation, which is $1163 \mathrm{r} / \mathrm{min}$. Figure 1 is a schematic diagram of the test bench. The impeller diameter of the shaft tubular pump is $300 \mathrm{~mm}$. Figure 2(a) shows a physical picture of the impeller. The number of blades is 3, and they are made of brass. The guide vanes and IGVs are welded with $5 \mathrm{~mm}$ thick steel materials. The guide vanes and IGVs are shown in Figures 2(b) and 2(c)2 (e). Three monitoring points, $P 1 *$ at the IGVs inlet, $P 2 *$ at the impeller inlet, and $P 3 *$ at the guide vane outlet, were measured in the pressure fluctuation test. Figure 2(f) is the physical map of the test device and the layout of the measuring points.

2.2. Test Results and Analysis. Figure 3 shows the test results of the external characteristics of pumps with 4 IGVs, 5 IGVs, and 6 IGVs. The change trends of the head and efficiency curves of the three schemes are basically the same. The number of IGVs has an obvious effect on the efficiency of the pump but has little effect on the head. When the number of IGVs gradually increases, the efficiency of the pump first increases and then decreases. The efficiency of the pump with $5 \mathrm{IGVs}$ is the highest under the design condition, which is approximately $1.26 \%$ higher than that of the shaft tubular pump with 4 IGVs.

To analyze the pressure pulsation, the nondimensional parameters of the pressure pulsation coefficient $C_{\mathrm{p}}$ and pressure pulsation intensity $C_{p}^{*}$ are introduced to express the results. The formulas are as follows:

$$
\begin{gathered}
C_{P}=\frac{P-\bar{P}}{0.5 \rho u^{2}}, \\
C_{P}{ }^{*}=\sqrt{\frac{\sum_{i=1}^{n} C_{P}^{2}}{n}},
\end{gathered}
$$

where $\bar{P}$ is the average pressure at the monitoring point, $\mathrm{Pa}$, $P$ is the instantaneous pressure at the monitoring point, $\mathrm{Pa}, \rho$ is the liquid density, $\mathrm{kg} / \mathrm{m}^{3}, n$ is the impeller revolution, and $u$ is the impeller outlet circumferential velocity, $\mathrm{rad} / \mathrm{s}$.

Figure 4 shows the test results for the pressure fluctuation of shaft tubular pumps with 4 IGVs, 5 IGVs, and 6 IGVs. Since the IGVs have an obvious influence on the pressure fluctuation at the impeller inlet, the following investigation mainly analyzes point $P 1 *$ at the impeller inlet. The figure shows that with increasing flow rate, the pressure fluctuation amplitude at the impeller inlet of the three schemes gradually decreases. When the number of IGVs gradually increases, the amplitude of pressure fluctuation at the impeller inlet first decreases and then increases under small-flow conditions and design conditions but gradually increases under large flow conditions. Under the design conditions, the pressure fluctuation amplitudes of the 4 IGVs and 6 IGVs shaft tubular pumps are approximately 2.6 times and 1.8 times that of the 5 IGVs shaft tubular pump, respectively. According to the results of the tests on the pressure fluctuation and external characteristics, the performance of the shaft tubular pump with $5 \mathrm{IGVs}$ is the best. Therefore, the following selection of the 5 IGVs scheme and no IGVs scheme shaft tubular pump are numerically calculated and compared. 


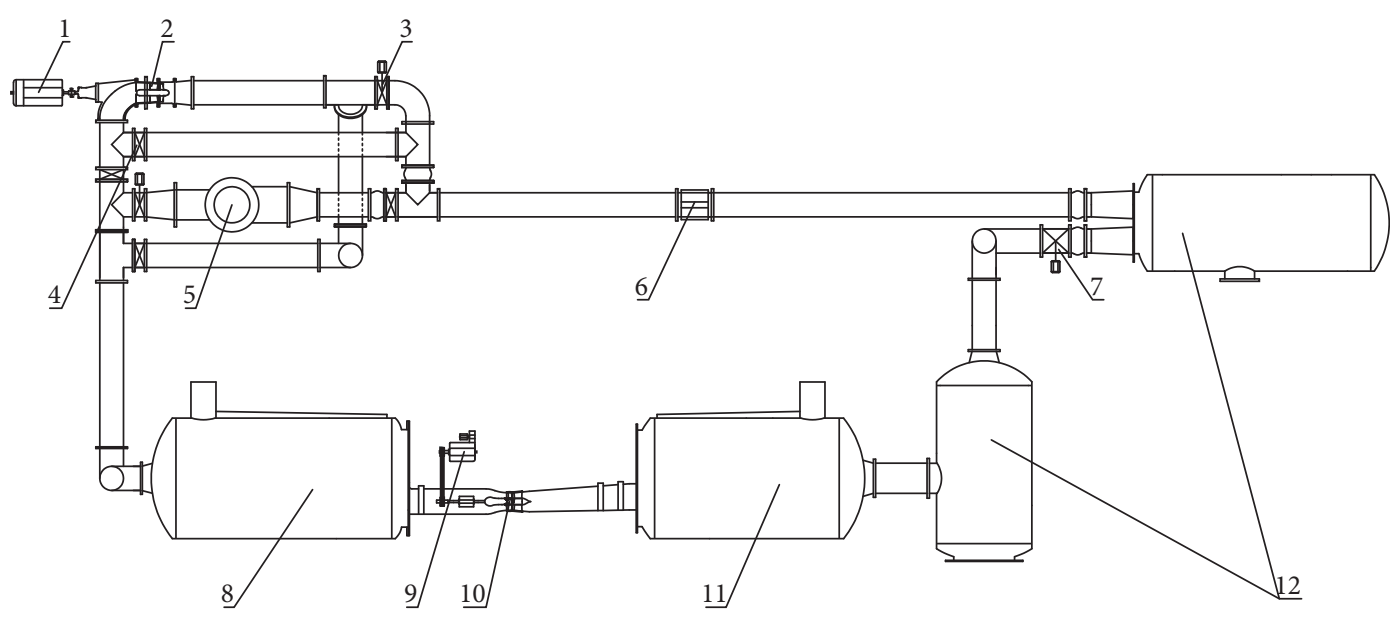

Figure 1: Two-dimensional diagram of the hydraulic machinery test bench (1: auxiliary pump motor; 2: auxiliary pump; 3: solenoid globe valve; 4: shut-off valve; 5: auxiliary pump; 6: electromagnetic flowmeter; 7: electric control gate valve; 8: vacuum water tank; 9: main motor; 10: shaft tubular pump device; 11: pressure water tank; and 12: water storage tank).

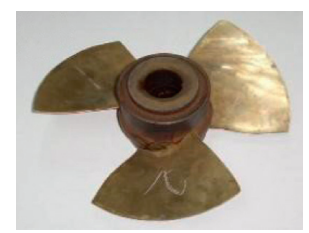

(a)

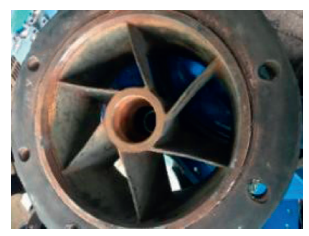

(b)

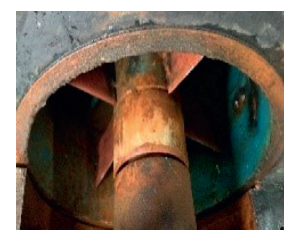

(c)

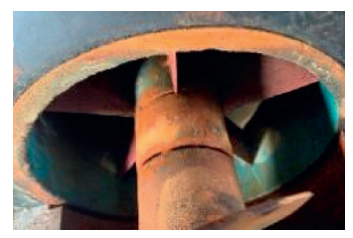

(d)

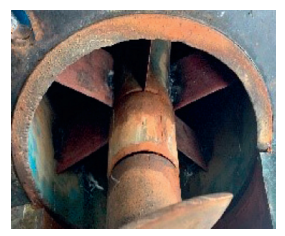

(e)

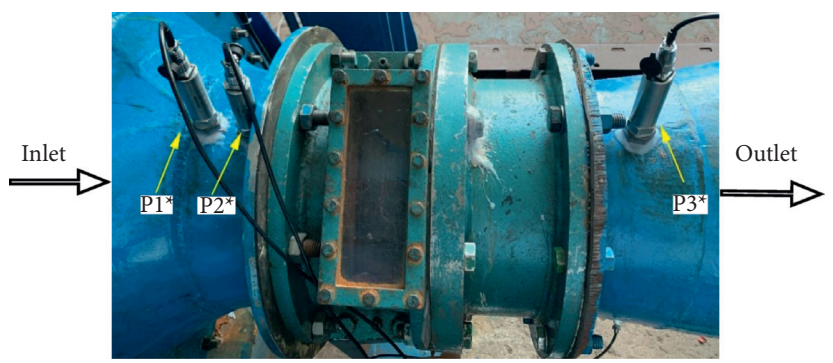

(f)

Figure 2: (a) Impeller, (b) guide vanes, (c) 4 IGVs, (d) 5 IGVs, (e) 6 IGVs, and (f) diagram of test device.

\section{Numerical Calculation}

3.1. Calculation Model. Numerical simulations of the 5 IGVs schemes and the scheme without IGVs are performed. The speed of the pump is $1163 \mathrm{r} / \mathrm{min}$. Under the design condition Qd, when the temperature is $25^{\circ} \mathrm{C}$, the Reynolds number of the inlet section of the impeller of the shaft tubular pump is 1510268. The hub diameter $D_{1}$ of the shaft tubular pump is $110 \mathrm{~mm}$, and the impeller diameter $D$ is $300 \mathrm{~mm}$. The pump has 3 impellers. The tip clearance $d$ is $0.15 \mathrm{~mm}$. The hub ratio $d_{r}$ is 0.4 . The design flow $Q_{\mathrm{d}}$ is $288 \mathrm{~L} / \mathrm{s}$. The single-sided spread angle of the guide vane shroud is 5 degrees. The IGVs adopt $2 \mathrm{D}$ a straight blade form. The installation angle of the IGVs is set to 0 degrees. The number of IGVs is 5 , and their thickness is $5 \mathrm{~mm}$. The distance between the IGVs and the impeller is set at $0.85 \mathrm{D}$. Figure 5 shows the three-dimensional model of the shaft tubular pump impeller.
The calculation model of the scheme without IGVs is composed only of impellers, guide vanes, inlet channels, and outlet channels. In addition to the above four parts, the calculation model of the IGVs scheme adds the IGVs section. The grids of the impeller, guide vanes, and IGVs are modeled by TurboGrid and divided into structured grids. The grid number of guide vanes is approximately 840,000. The grid number of IGVs is approximately 960,000. The inlet and outlet flow channels are first modeled by Creo and then divided into structured grids using ICEM. The numbers of grid elements are approximately 1.41 million and 710,000. The mesh of each part of the model is shown in Figure 6. After the mesh of each part is completed, the calculation model is assembled in CFX. The three-dimensional diagrams of the calculation model are shown in Figure 7.

Because the convergence of the calculation is greatly affected by the grid quality, grid-independence analysis of 


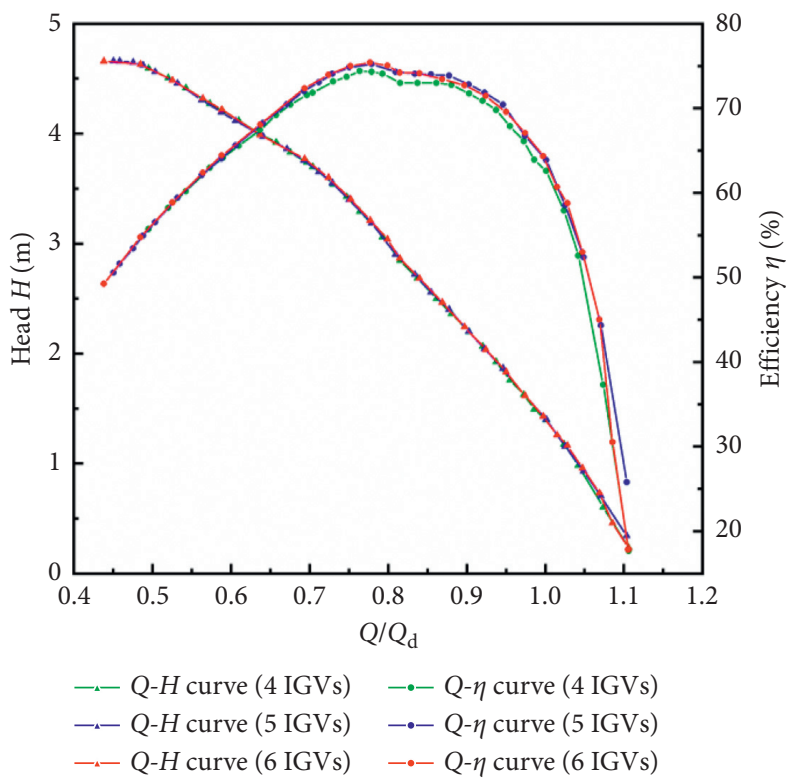

FIgURE 3: Test results for shaft tubular pumps with 4, 5, and 6 IGVs.

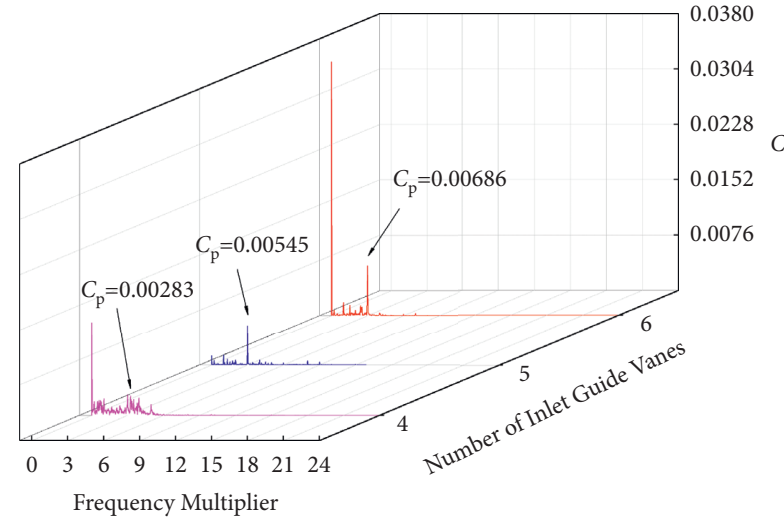

(a)

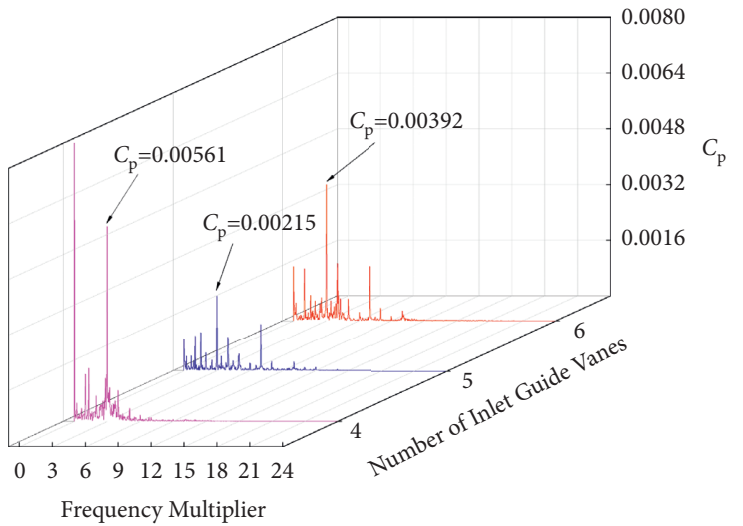

(b)

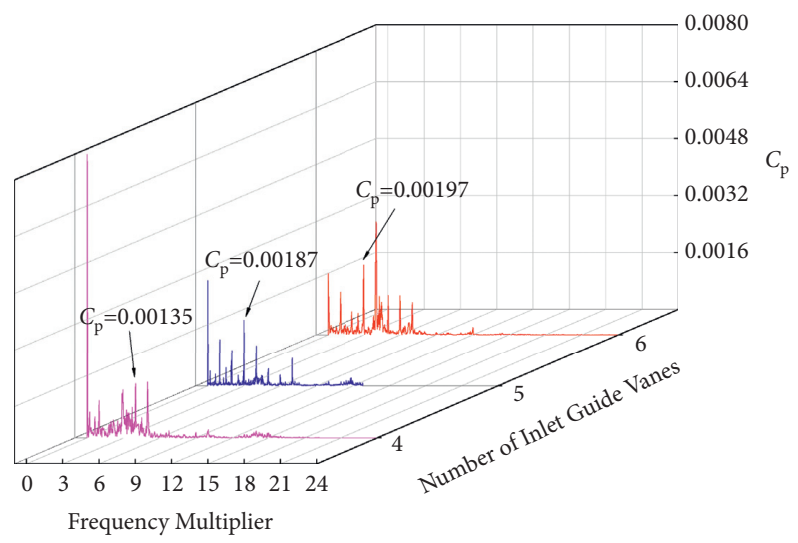

(c)

Figure 4: Test results for the pressure pulsation test of shaft tubular pumps with 4, 5, and 6 IGVs: (a) $0.75 Q_{d}$ and (b) $Q_{d}$ and $1.25 Q_{d}$. 


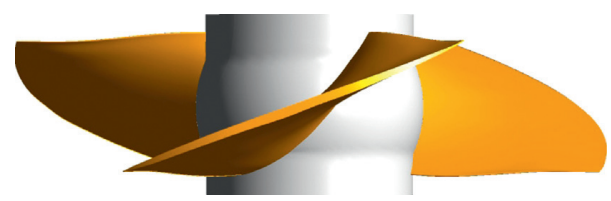

FIgURE 5: Three-dimensional model of the impeller.

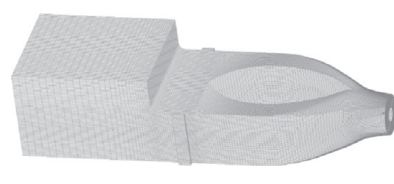

(a)

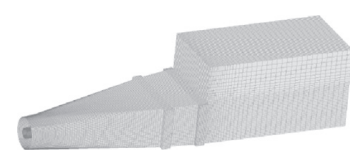

(b)

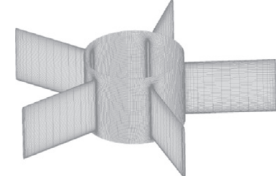

(c)

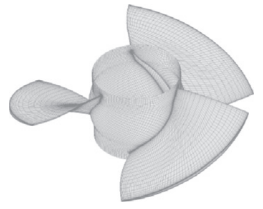

(d)

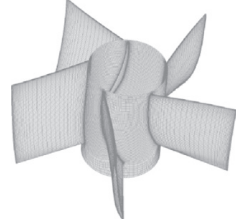

(e)

FIGURE 6: Grid of each part. (a) Inlet channel. (b) Outlet flow channel. (c) IGVs. (d) Impeller. (e) Guide vanes.

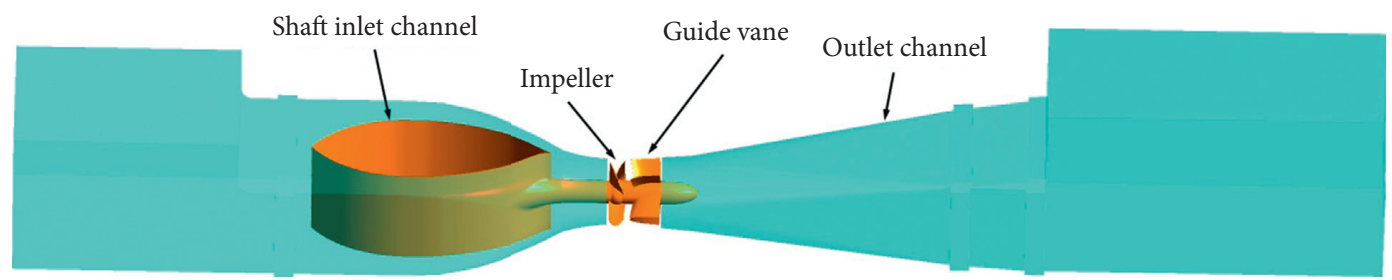

(a)

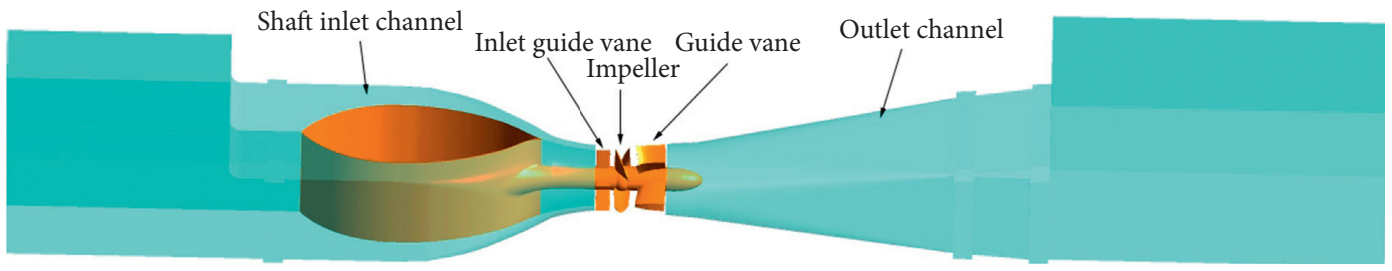

(b)

FIgURE 7: Three-dimensional diagram of the calculation model of the pump device (a). Scheme of the shaft tubular pump without IGVs (b). Scheme of the shaft tubular pump with IGVs.

the shaft tubular pump with and without IGVs is performed. Figure 8 shows the relationship between the grid element number and the efficiency of the pump device.

When the number of grid elements for the pump device without and with IGVs is 3.2 million and 4 million, respectively, the efficiency is basically no longer affected by changes in the grid. To reduce the workload of the numerical calculations, a total number of grid elements of the pump device without and with IGVs are selected to be 3.43 million and 4.4 million, respectively.
3.2. Governing Equation. The basic governing equation in this article is the N-S equation. The turbulence model uses RNG $k-\varepsilon$ turbulence model. The $k-\varepsilon$ turbulence model is a common calculation model suitable for most projects. The RNG $k-\varepsilon$ turbulence model is an improved equation based on the standard $k-\varepsilon$ turbulence model, which can deal with swirling and large aberration flows, so it is more widely used.

(1) The $k$ equation and $\varepsilon$ equation of the RNG $k$ - $\varepsilon$ turbulence model are

$$
\begin{aligned}
& \frac{\partial(\rho k)}{\partial(t)}+\frac{\partial\left(\rho k u_{i}\right)}{\partial\left(x_{i}\right)}=\frac{\partial}{\partial x_{j}}\left[\alpha_{k} \mu_{e f f} \frac{\partial_{k}}{\partial x_{j}}\right]+\mu_{t}\left(\frac{\partial u_{i}}{\partial x_{j}}+\frac{\partial u_{j}}{\partial x_{i}}\right) \frac{\partial u_{i}}{\partial x_{j}}-\rho \varepsilon \\
& \frac{\partial(\rho \varepsilon)}{\partial(t)}+\frac{\partial\left(\rho \varepsilon u_{i}\right)}{\partial\left(x_{i}\right)}=\frac{\partial}{\partial x_{j}}\left[\alpha_{\varepsilon} \mu_{e f f} \frac{\partial_{\varepsilon}}{\partial x_{j}}\right]+\frac{C_{1 \varepsilon} \cdot \varepsilon}{k} \mu_{t}\left(\frac{\partial u_{i}}{\partial x_{j}}+\frac{\partial u_{j}}{\partial x_{i}}\right) \frac{\partial u_{i}}{\partial x_{j}}-C_{2 \varepsilon \rho} \frac{\varepsilon^{2}}{k}-R
\end{aligned}
$$




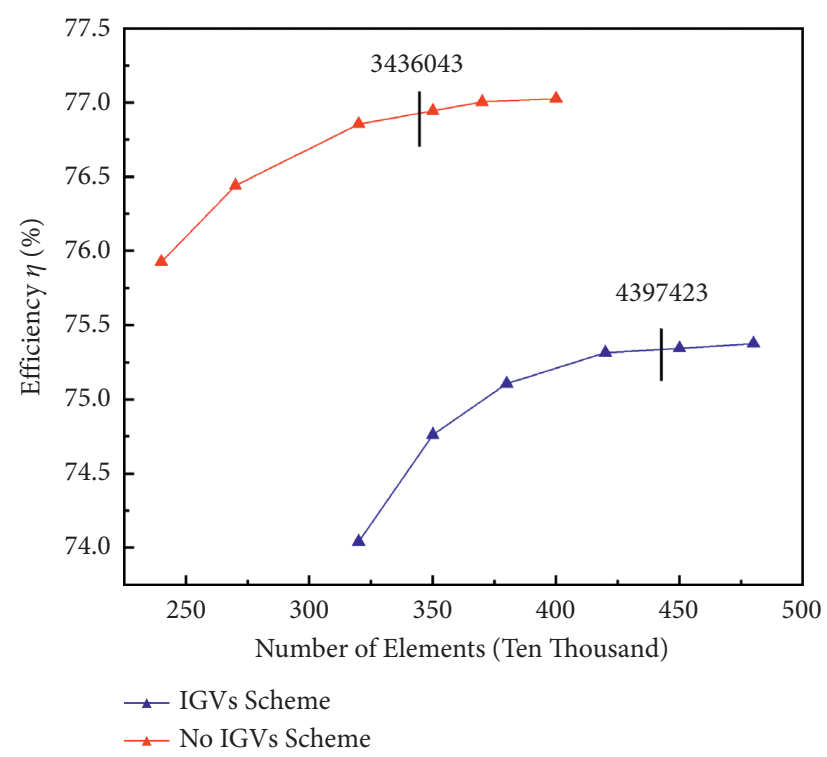

FIgURE 8: Grid-independence analysis.

where $\rho$ is the fluid density, $x_{i}$ and $x_{j}$ are the coordinate components, $\mu_{t}$ is the turbulent viscosity, $u_{i}$ and $u_{j}$ are the time-averaged velocity components, $\alpha_{k}$ and $\alpha_{\varepsilon}$ are the corresponding coefficients of $k$ and $\varepsilon$, respectively, and $\mu_{\text {eff }}$ is the effective turbulent flow viscosity coefficient:

$$
\begin{aligned}
\mu_{e f f} & =\mu+\mu_{t} ; \\
\mu_{t} & =\rho C_{\mu} \frac{k^{2}}{\varepsilon} ; \\
R & =\frac{C_{\mu} \rho \varphi^{3}\left(1-\varphi / \varphi_{0}\right) \varepsilon^{2}}{\left(1+\beta \varphi^{3}\right) k} ; \\
C_{\mu} & =0.0845 ; \\
C_{1 \varepsilon} & =1.42 ; \\
C_{2 \varepsilon} & =1.68 ; \\
\varphi & =\left(2 E_{i j}\right) \frac{k}{3} ; \\
E_{i j} & =\frac{1}{2}\left(\frac{\partial u_{i}}{\partial x_{i}}+\frac{\partial u_{j}}{\partial x_{j}}\right) ; \\
\varphi_{0} & =4.377 ; \\
\beta & =0.012 .
\end{aligned}
$$

(2) The three-dimensional incompressible turbulent continuity equation and momentum equation are

$$
\begin{aligned}
\frac{\partial \rho}{\partial t}+\nabla \cdot(\rho \bar{U}) & =0 \\
\frac{\partial \rho \bar{U}}{\partial t}+\nabla \cdot\left(\rho \bar{U} \cdot U^{\prime}\right)-\nabla \cdot\left(\mu_{e f f} \nabla \cdot U^{\prime}\right) & =\nabla P^{\prime}+\nabla \cdot\left(\mu_{e f f} \nabla \bar{U}\right)^{T}+\bar{B},
\end{aligned}
$$

where $P^{\prime}=P+2 / 3 \rho K$ is the static pressure, $\mu^{\prime}$ is the velocity component, and $\bar{B}$ is the sum of additional forces.

\subsection{Boundary Condition and Pressure Fluctuation Monitoring} Points. The governing equation is the N-S equation in the unsteady numerical simulation of the pump device. The RNG $k-\varepsilon$ turbulence model is a basic turbulence model $[42,43]$. The inlet boundary condition is set as the total pressure inlet, and its pressure value is one atmosphere. The outlet boundary condition is set as the mass flow outlet. The impeller is set as rotating domain. The other parts are set as static domain. The nonslip condition satisfying viscous fluid is adopted. The dynamic and static interface adopts the stage interface model with average speed. The other parts of the interface adopt the none interface type $[44,45]$. The time step is calculated and set to $4.7619 \times 10^{-4} \mathrm{~s}$. The total time step is the time required to rotate 8 revolutions, which is set to $0.45714286 \mathrm{~s}$. To verify the independence of the time step, two time steps of $1.5873 \times 10^{-4} \mathrm{~s}$ (equivalent to 1 degree impeller rotation time) and $3.174610 \times 10^{-4} \mathrm{~s}$ (equivalent to 2 degree impeller rotation time) are calculated for the tubular pump without IGVs. We extract the pressure pulsation at point $\mathrm{P} 5$ at the impeller inlet under the design condition $Q_{\mathrm{d}}$ for comparison, as shown in Figure 9. The difference in pressure pulsation calculated by the three time steps is 


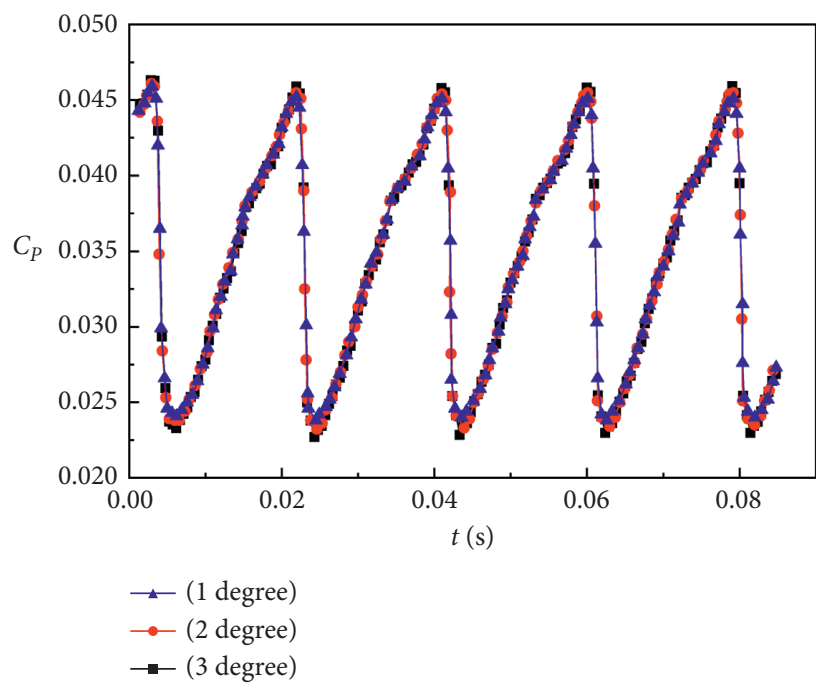

FIGURE 9: Independence of the time step.

relatively small. Therefore, to reduce the calculation time, it is reasonable to select 3 degrees as a time step for calculation.

We arrange monitoring points in the shaft tubular pump to analyze the pressure fluctuation characteristics. The P1-P4 monitoring points are arranged radially on the IGVs inlet section. The P5-P8 monitoring points are arranged radially on the impeller inlet section. The P9-P12 monitoring points are arranged radially on the impeller outlet section. The P13P16 monitoring points are arranged radially on the guide vane outlet section. The three-dimensional layout of monitoring points is shown in Figure 10.

\section{Comparison of the Results of the Calculation and the Experiment}

4.1. External Characteristic Results. The model test results for the shaft tubular pump with and without IGVs are compared with the calculation results. Figure 11 shows the comparison result. The figure shows that when the operating condition deviates from the design condition, the efficiency measured by the model test is higher than that of the calculation. When the flow is higher than the design flow conditions, the head curve fits better. The head deviation is larger when it is less than the design flow, but the maximum relative error does not exceed 5\%. This shows that the numerical simulation results of the shaft tubular pump are accurate and credible. This also demonstrates that the performance of the shaft tubular pump will be significantly affected by the IGVs.

4.2. Pressure Pulsation Results. The model test also tested the pressure pulsation at point $P 1 *$ under three characteristic working conditions $\left(0.75 Q_{\mathrm{d}}, Q_{\mathrm{d}}\right.$, and $\left.1.25 Q_{\mathrm{d}}\right)$ of the shaft tubular pump with 5 IGVs and no IGVs. Figures 12 and 13 show the comparison between the pressure pulsation test and the numerical calculation results. The main frequency of the pressure fluctuation of the two schemes is the impeller passing frequency, and the amplitude deviation is small. The calculation results of the pressure fluctuation are shown to be accurate and reliable.

\section{Numerical Results}

5.1. Analysis of the Hydraulic Performance. To analyze the performance difference of the shaft tubular pump without IGVs and with 5 IGVs schemes, numerical simulation calculations are performed on the two pump devices. Figure 14 shows the comparison results of the performance curves. After adding the IGVs in front of the impeller, the pump head and efficiency are reduced under design conditions and small-flow conditions. The smaller the flow is, the greater the drop in the head and efficiency of the shaft tubular pump device are. However, there is basically no change in the head and efficiency under large-flow conditions. Under the design condition, the efficiency of the pump with IGVs is nearly $1.63 \%$ lower than that of the scheme without the IGVs, and the head is nearly $0.06 \mathrm{~m}$ lower. The IGVs have a greater impact on the performance of the shaft tubular pump.

To further analyze the influence of the IGVs, Figure 15 is the comparison of the flow uniformity $V_{\mathrm{u}}$ and the speed weighted average angle $\theta$ of the impeller inlet of the two pump schemes. With increasing flow, the uniformity of the flow velocity and the velocity-weighted average angle of the impeller inlet section of the two schemes gradually increase. After adding the IGVs in front of the impeller, the flow uniformity of the impeller inlet section increases by approximately $1.11 \%$, and the speed-weighted average angle is reduced by approximately $0.7^{\circ}$. This shows that the IGVs can improve the hydraulic conditions of the impeller of the shaft tubular pump:

$$
V_{u}=\left(1-\frac{1}{V_{a}} \sqrt{\frac{\sum_{i=1}^{n}\left(V_{a i}-V_{a}\right)^{2}}{m}}\right) \times 100 \%,
$$




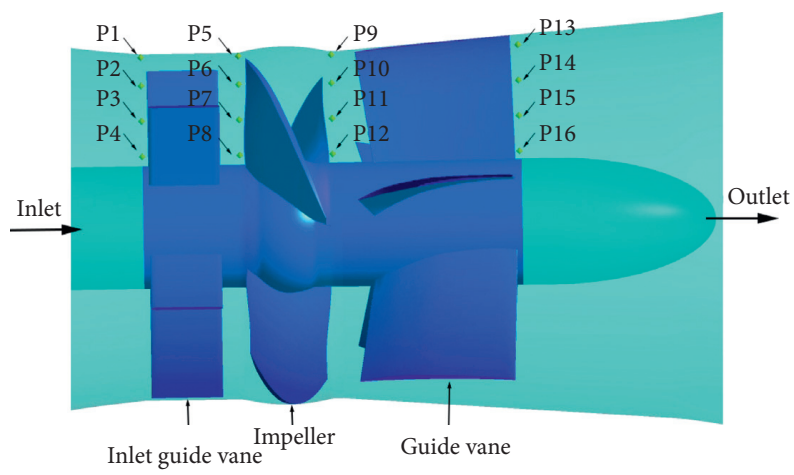

FiguRE 10: Three-dimensional schematic diagram of the layout of monitoring points.

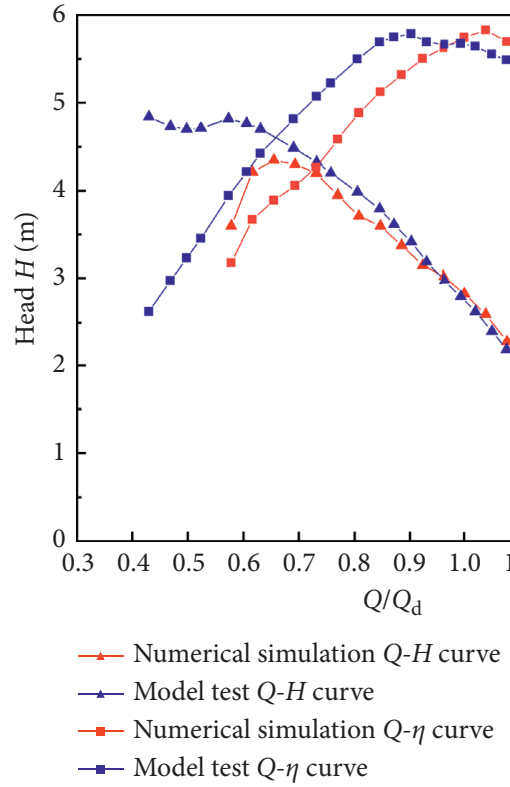

(a)

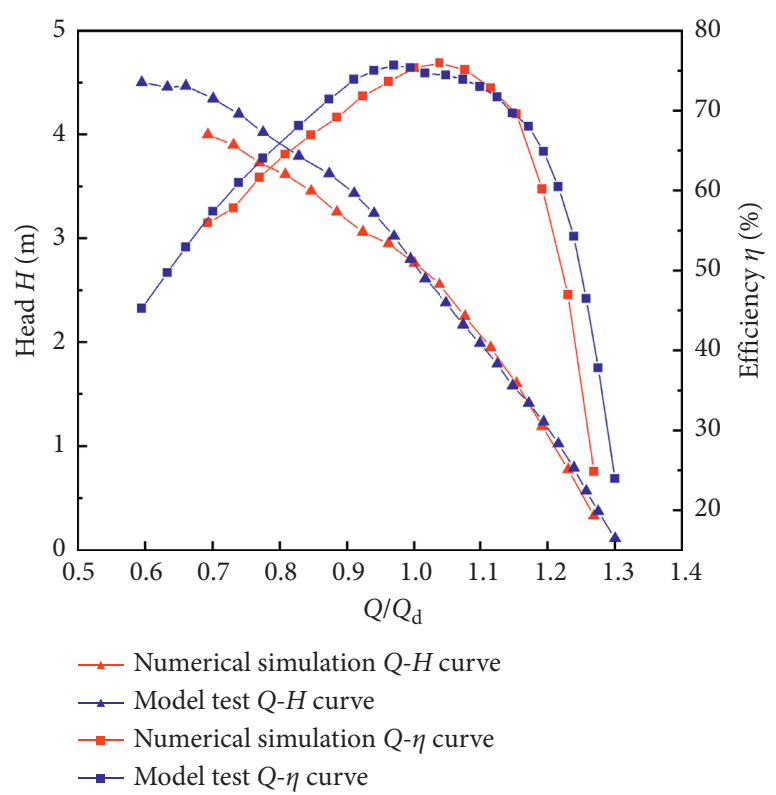

(b)

FIGURE 11: Comparison between the results of the calculation and the test. (a) Shaft tubular pump without IGVs and (b) shaft tubular pump with IGVs.
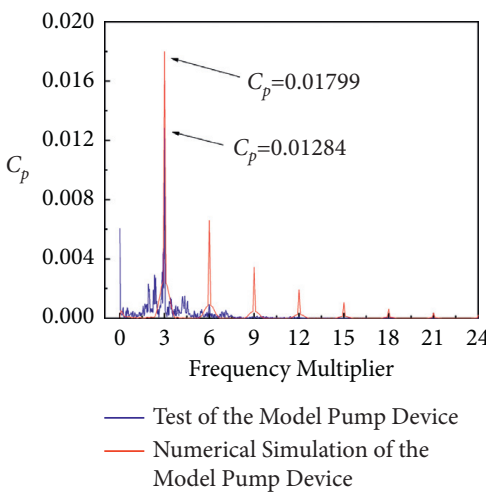

(a)
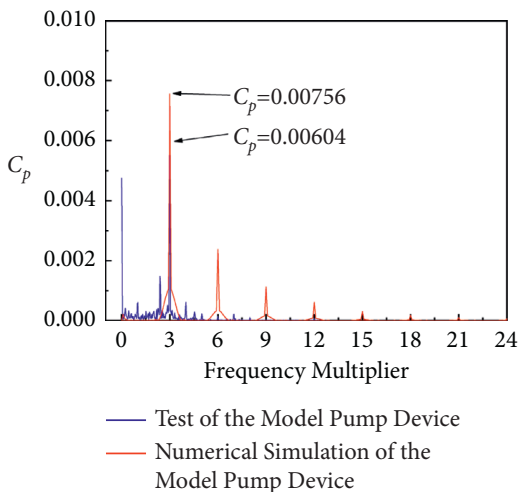

(b)

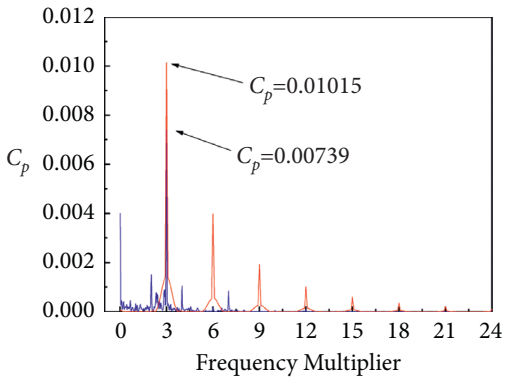

— Test of the Model Pump Device

Numerical Simulation of the Model Pump Device

(c)

FIGURE 12: Test of the pressure fluctuation and numerical simulation comparison of impeller inlet of shaft tubular pumps with no IGVs under (a) $0.75 Q_{\mathrm{d}}$, (b) $Q_{\mathrm{d}}$, and (c) $1.25 Q_{\mathrm{d}}$. 


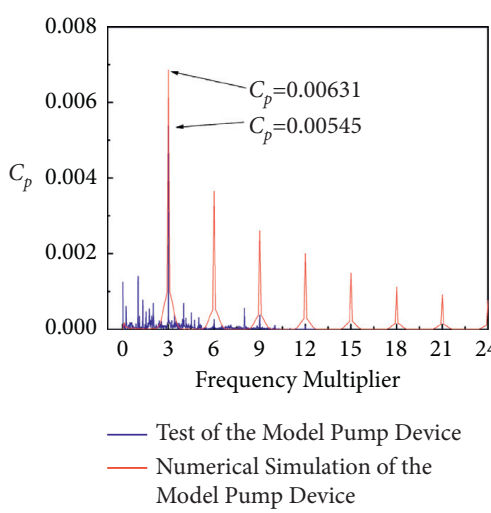

(a)

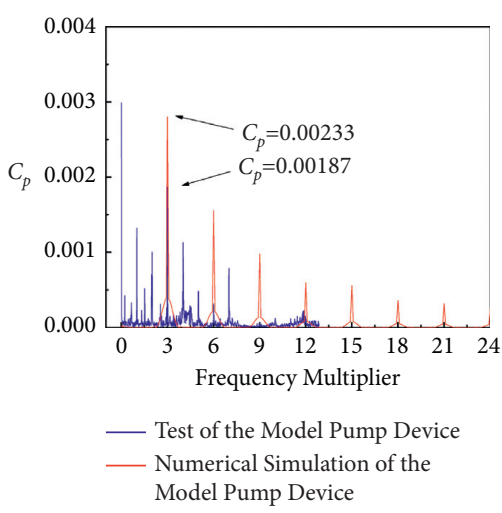

(b)

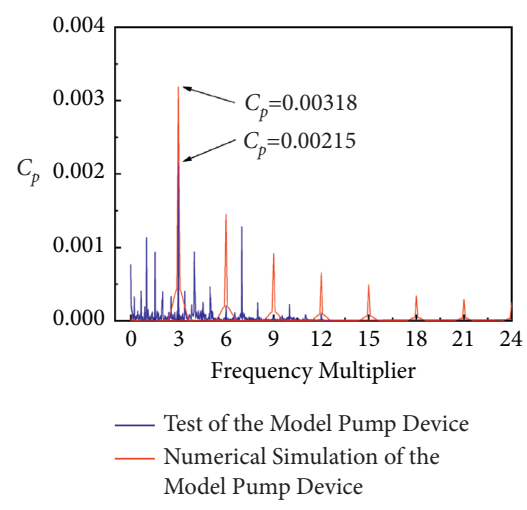

(c)

FIGURE 13: Test of the pressure fluctuation and numerical simulation comparison of the impeller inlet of shaft tubular pumps for the 5 IGVs scheme under (a) $0.75 Q_{\mathrm{d}}$, (b) $Q_{\mathrm{d}}$, and (c) $1.25 Q_{\mathrm{d}}$.

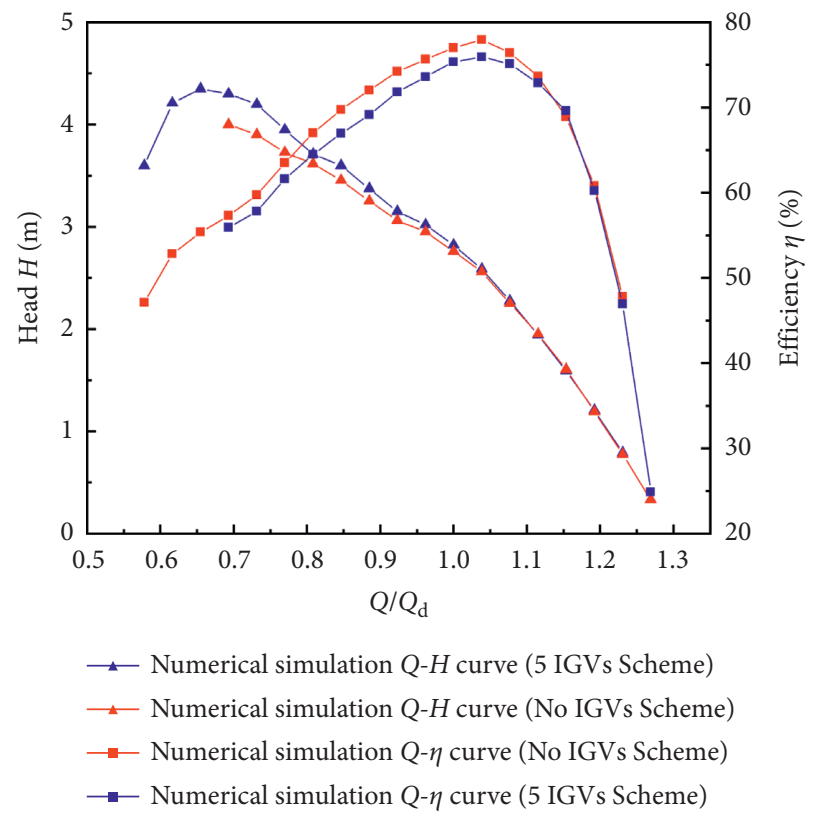

FIgURE 14: External characteristic curve of the numerical simulation of the two schemes.

where $V_{a i}$ is the axial velocity of the $i$ th grid node of the impeller inlet section, $\mathrm{m} / \mathrm{s}, V_{a}$ is the average axial velocity of the impeller inlet section, $\mathrm{m} / \mathrm{s}$, and $m$ is the number of grids of the impeller inlet section:

$$
\theta=\frac{\sum_{i=1}^{n}\left(90^{\circ}-\arctan \left(V_{\mathrm{ti}} / V_{\mathrm{ai}}\right)\right) V_{\mathrm{ai}}}{\sum_{i=1}^{n} V_{\mathrm{ai}}}
$$

where $V_{t i}$ is the lateral velocity of the $i$ th grid node on the impeller inlet section, $\mathrm{m} / \mathrm{s}$.

Figure 16 shows the change in the static pressure of each calculation area in the shaft tubular pump with and without IGVs under three characteristic working conditions $(0.75$ $Q_{\mathrm{d}}, Q_{\mathrm{d}}$, and $\left.1.25 Q_{\mathrm{d}}\right) . \Delta H$ is the average static pressure difference between each section and the impeller inlet. $Z$ is the distance from the section to the central section of the impeller [46]. After adding the IGVs in front of the impeller under various working conditions, $\triangle H$ of the inlet flow passage and the IGVs section significantly decrease. As the flow rate increases, $\triangle H$ decreases more, but the values of $\triangle H$ of the impeller, guide vanes, and outlet channel basically do not change. It can be found from the results that the hydraulic loss of the inlet channel will increase by adding the IGVs, and the performance of the shaft tubular pump will decrease.

The axial velocity diagrams of the impeller inlet section of the shaft tubular pump under three characteristic conditions $\left(0.75 Q_{\mathrm{d}}, Q_{\mathrm{d}}\right.$, and $\left.1.25 \mathrm{Q}_{\mathrm{d}}\right)$ are considered for comparison, as shown in Figures 17 and 18. The figures show that due to the influence of the impeller, three high-velocity areas and three low-velocity areas appear on the inlet of the impeller of the two schemes under three working conditions, 


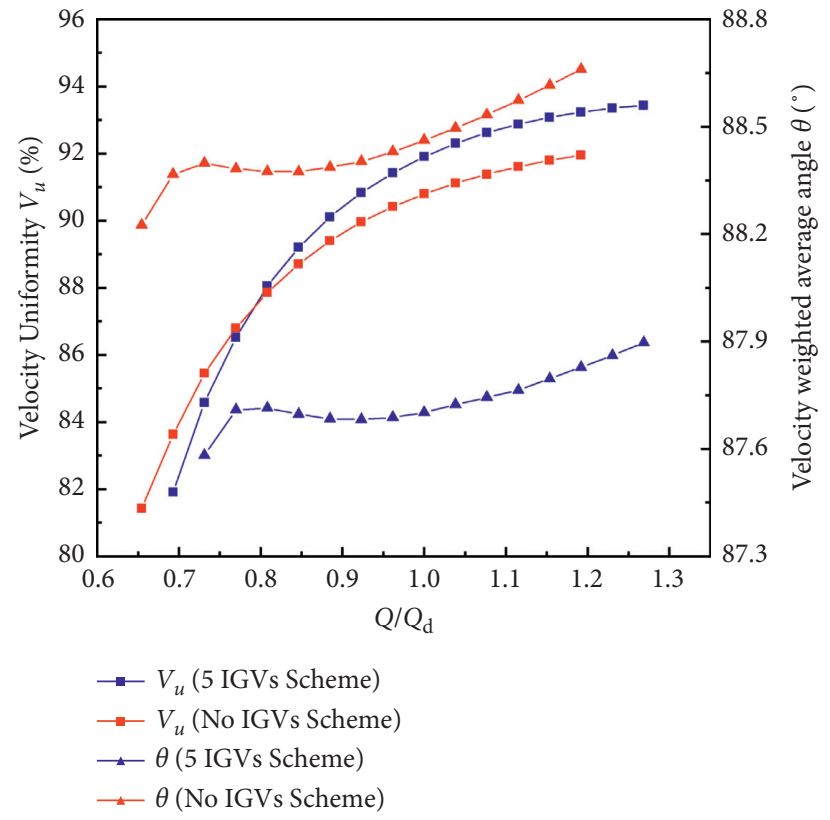

FIGURE 15: Comparison of the uniformity of the flow velocity and velocity-weighted average angle at the impeller inlet.

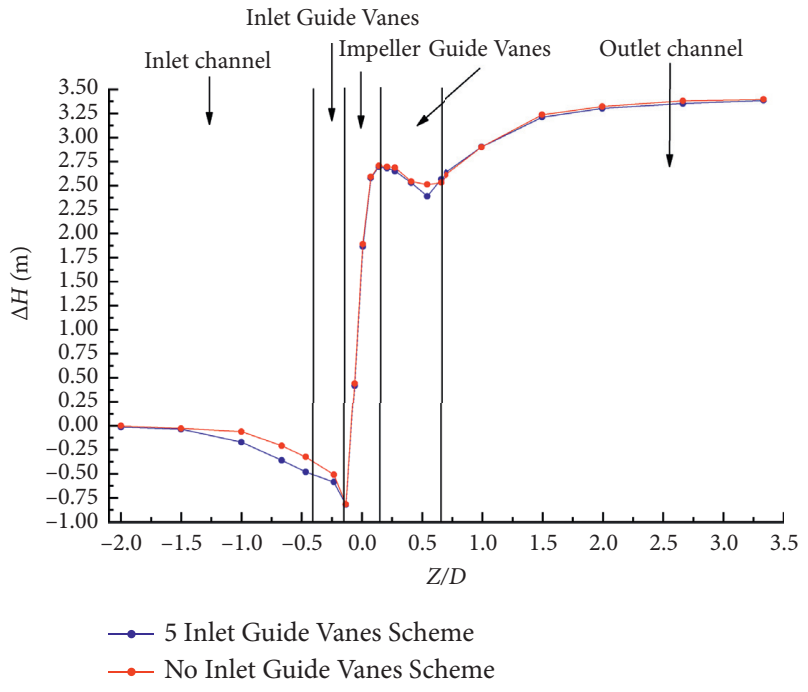

(a)

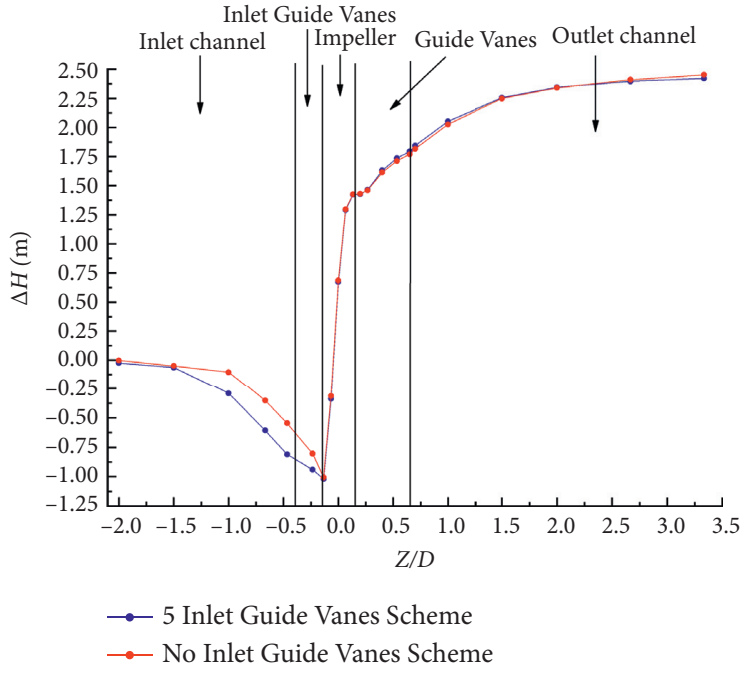

(b)

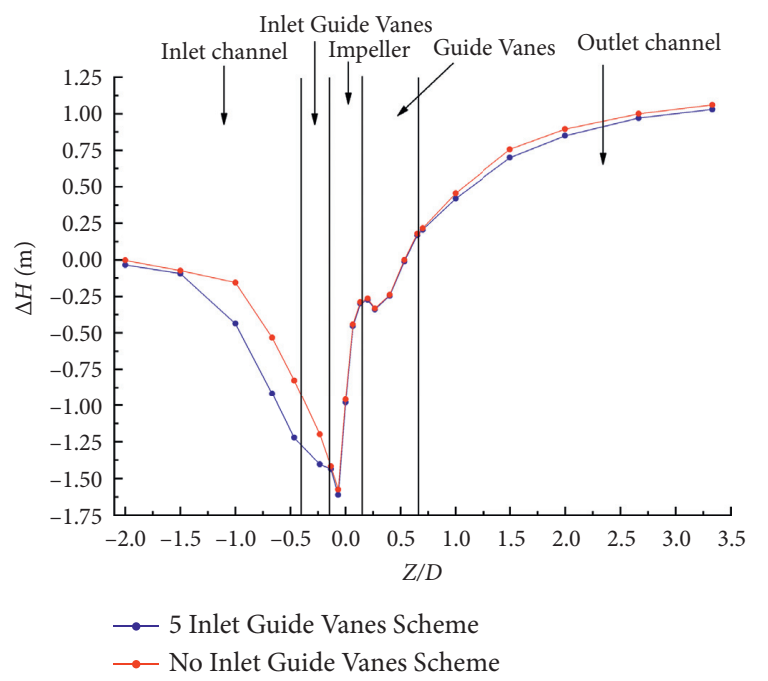

(c)

Figure 16: Hydraulic curves of each calculation area of the shaft tubular pump under (a) $0.75 Q_{d}$, (b) $Q_{d}$, and (c) $1.25 Q_{d}$. 

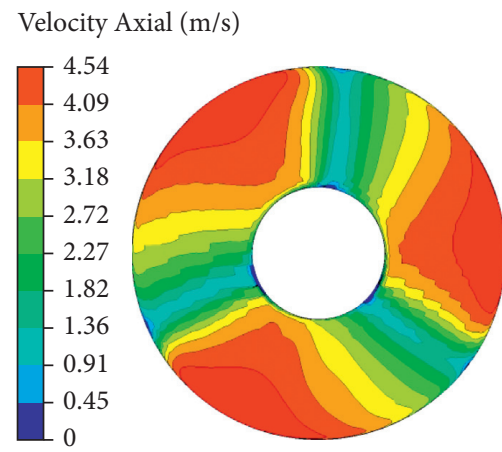

(a)
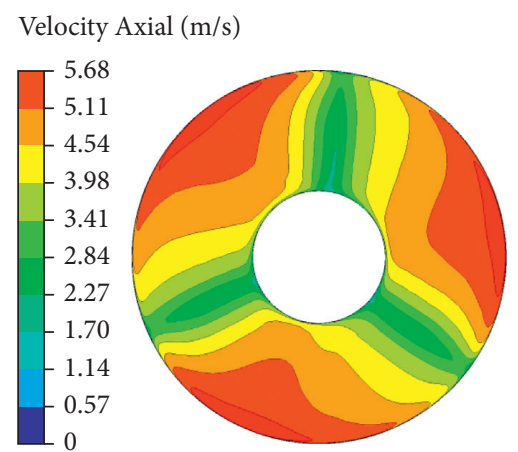

(b)
Velocity Axial (m/s)
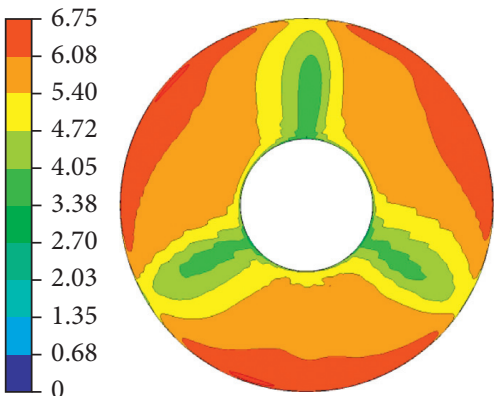

(c)

FIgURe 17: Axial velocity distribution of the pump impeller inlet in the no IGVs scheme (a) $0.75 Q_{\mathrm{d}}$, (b) $Q_{\mathrm{d}}$, and (c) $1.25 Q_{\mathrm{d}}$.
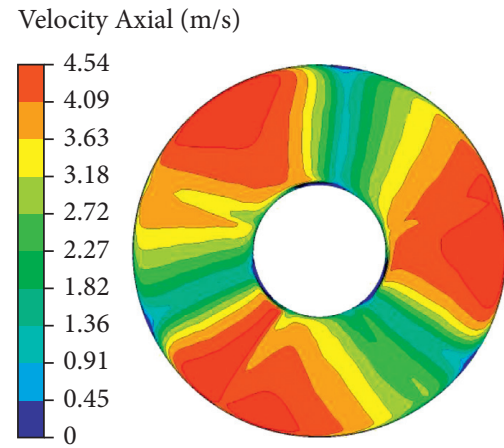

(a)
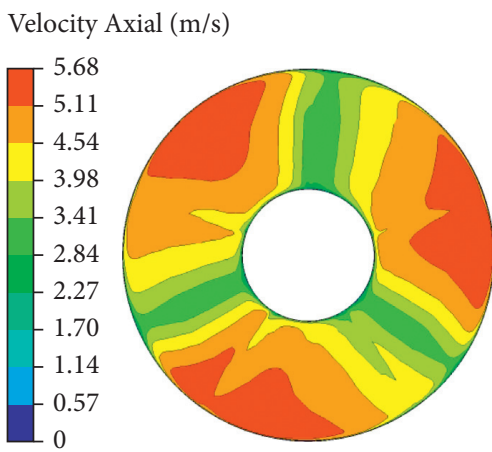

(b)
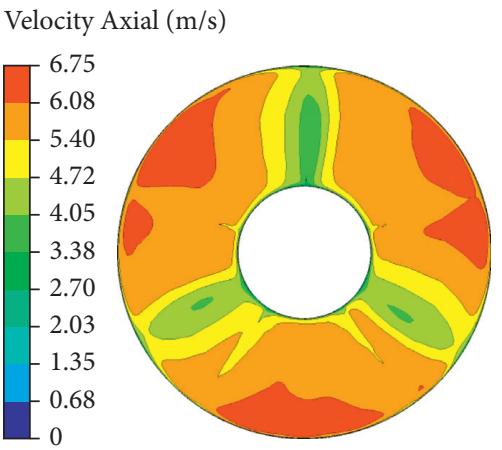

(c)

FIGURE 18: Axial velocity distribution of the pump impeller inlet in the 5 IGVs scheme under (a) $0.75 Q_{d}$, (b) $Q_{d}$, and (c) $1.25 Q_{d}$.

which are consistent with the number of blades. After adding the IGVs, the axial velocity decreases under three working conditions. This is because of the thickness of the IGVs and the frictional resistance of the contact surface between the blade and the water flow. It has an obstructive effect on the water flow, resulting in a decrease in the axial velocity of the water flow at the inlet of the impeller. There is frictional resistance between the IGVs and the water flow, which causes a decrease in the axial velocity of the impeller.

5.2. Pressure Pulsation Analysis. To analyze the difference between the pressure pulsation in the shaft tubular pump of the two schemes, the main frequency amplitude of pressure pulsation in the shaft tubular pump under three characteristic conditions $\left(0.75 Q_{\mathrm{d}}, Q_{\mathrm{d}}\right.$, and $\left.1.25 Q_{\mathrm{d}}\right)$ is taken for comparison, as shown in Figure 19. The inlet of the impeller is the area with the largest amplitude of pressure fluctuation, and the second is the impeller outlet. Because the inlet channel and the guide vanes are far from the impeller, the pressure fluctuation amplitude is small. The pressure pulsation amplitude of the shaft tubular pumps of the two schemes under small-flow conditions is the smallest under the three conditions. The pressure fluctuation amplitude under the design condition is the smallest, which is approximately $2 / 5$ of that under the small-flow condition. Therefore, more attention should be given to pressure pulsation under the condition of small flow when the shaft tubular pump is running. After the IGVs are added to the impeller inlet, the pressure pulsation of the entire pump device is reduced under each flow condition, especially the pressure pulsation of the impeller inlet, which greatly decreases. This shows that the IGVs can not only improve the uniformity of the impeller inlet flow rate but also suppress the pressure pulsation at the inlet of the impeller. After the IGVs are added, the pressure fluctuation amplitude in the inlet channel and the guide vanes is also very small. This is because the inlet channel and the guide vanes are far from the impeller and are less affected by the impeller.

The pressure pulsation intensity $C_{p}^{*}$ distribution of the impeller inlet section of the pump under three conditions $\left(0.75 Q_{\mathrm{d}}, Q_{\mathrm{d}}\right.$, and $\left.1.25 Q_{\mathrm{d}}\right)$ is taken for comparison, as shown in Figures 20 and 21. The figures show that there are 3 highpressure areas and 3 low-pressure areas at the inlet section of the impeller of the two schemes, which correspond to the number and position of impellers. This is because the rotation of the impeller is the source of pressure fluctuation inside the pump unit. After adding the IGVs, the pressure pulsation intensity at the entrance of the impeller will be greatly reduced under all working conditions. 


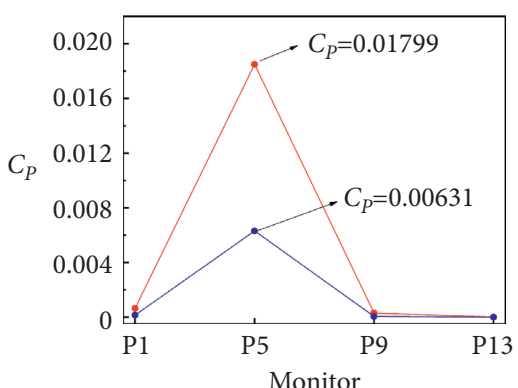

$\therefore$ No Inlet Guide Vanes Scheme $\rightarrow 5$ Inlet Guide Vanes Scheme

(a)

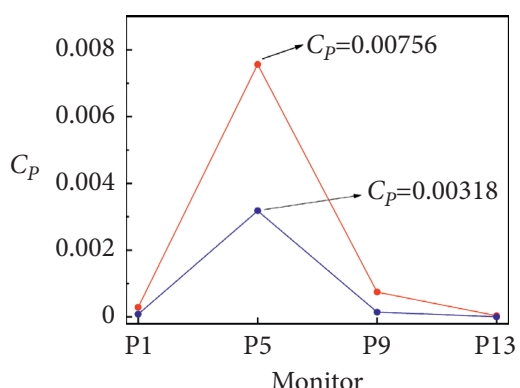

$\rightarrow$ No Inlet Guide Vanes Scheme $\rightarrow 5$ Inlet Guide Vanes Scheme

(b)

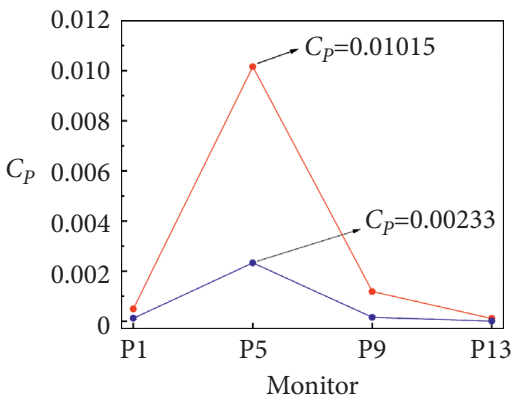

$\rightarrow$ No Inlet Guide Vanes Scheme $\rightarrow 5$ Inlet Guide Vanes Scheme

(c)

Figure 19: Main frequency of pressure pulsation in the shaft tubular pump under (a) $0.75 Q_{\mathrm{d}}$, (b) $Q_{\mathrm{d}}$, and (c) $1.25 Q_{\mathrm{d}}$.

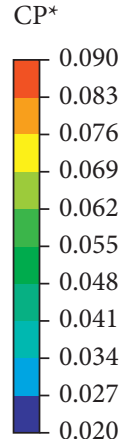

020

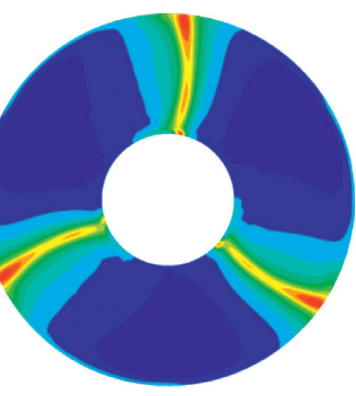

(a)

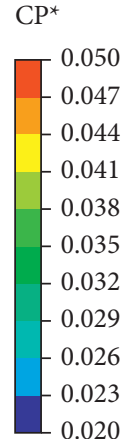

0.020

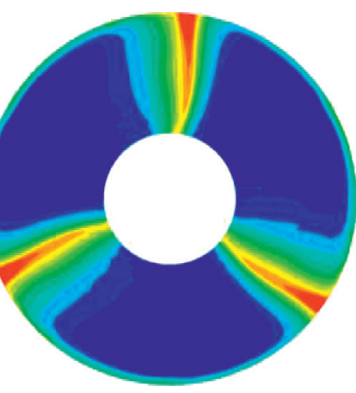

(b)

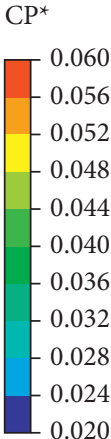

0.020

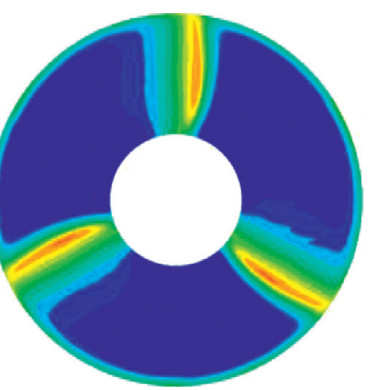

(c)

Figure 20: Pressure fluctuation intensity distribution of the pump impeller inlet in the no IGVs scheme under (a) $0.75 Q_{d}$, (b) $Q_{d}$, and (c) $1.25 Q_{\mathrm{d}}$.

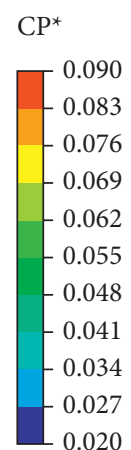

0.020

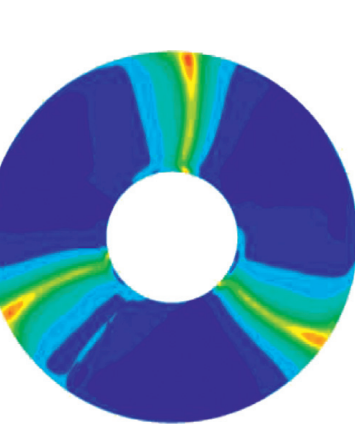

(a)

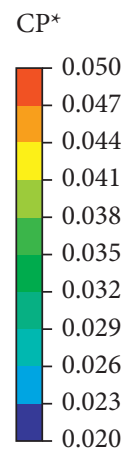

0.020

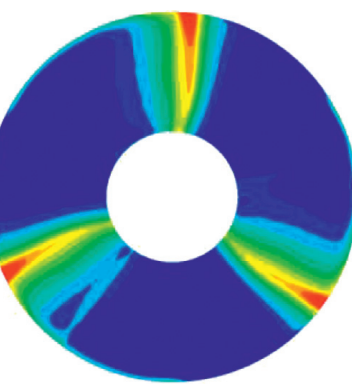

(b)

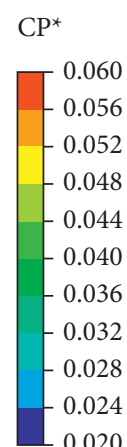

0.020

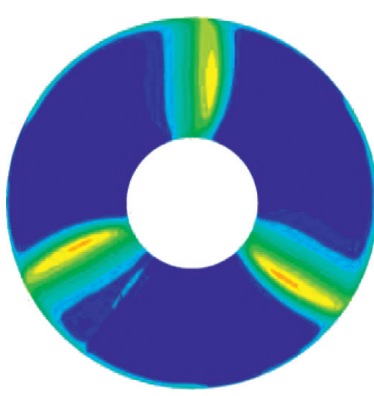

(c)

Figure 21: Pressure fluctuation intensity distribution of the pump impeller inlet in the 5 IGVs scheme under (a) $0.75 Q_{d}$, (b) $Q_{d}$, and (c) $1.25 Q_{\mathrm{d}}$.

To analyze the pressure fluctuation intensity distribution on the blade surface, the middle span circumferential section ( $\operatorname{span}=0.5$ ) of the hub and shroud of the impeller was removed for analysis. The midspan surface is shown in Figure 22. Figure 23 shows the pressure pulsation intensity distribution at the midspan. The pressure fluctuation intensity on the pressure side of the two schemes is higher than that on the suction side. The existence of IGVs reduces the pressure fluctuation intensity on the blade surface. With the decrease in the flow, the effect becomes increasingly obvious. After adding the IGVs, the pressure pulsation amplitude of the pressure side and suction side of 


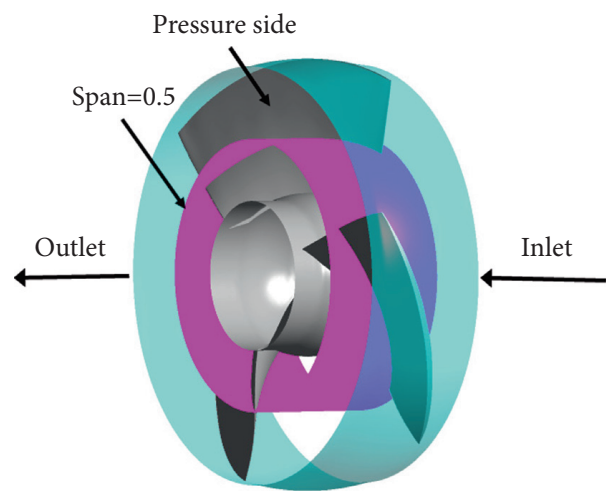

FIGURE 22: Schematic diagram of the midspan of the impeller.

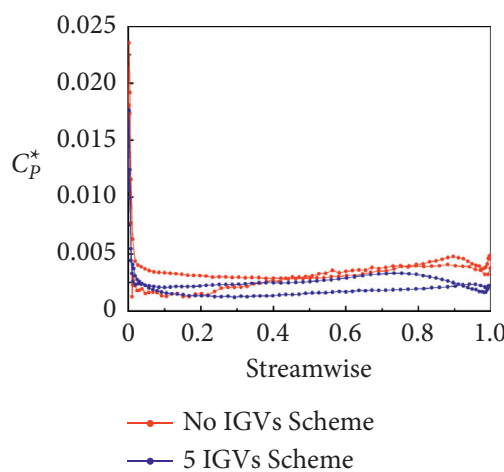

(a)

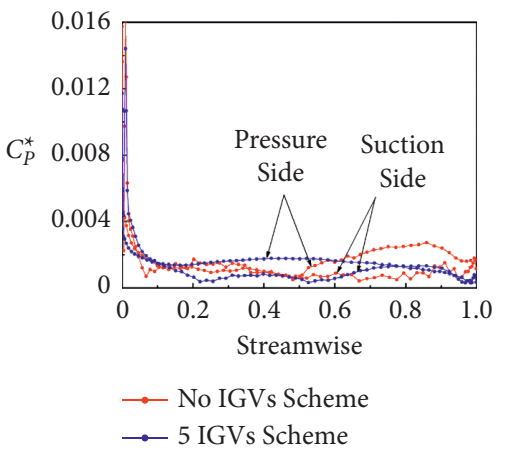

(b)

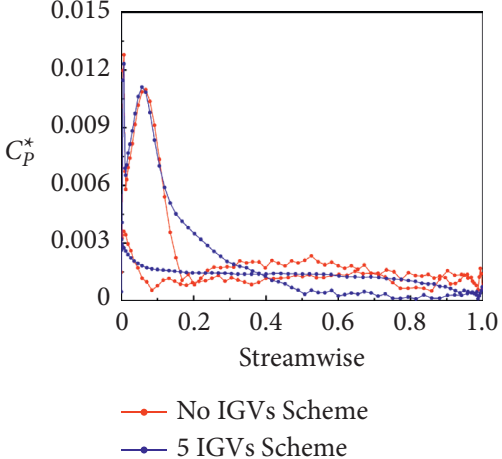

(c)

FIgURE 23: The pressure distribution of the suction surface and pressure surface of the blade in the midspan under (a) $0.75 Q_{d}$, (b) $Q_{d}$, and (c) $1.25 Q_{\mathrm{d}}$.

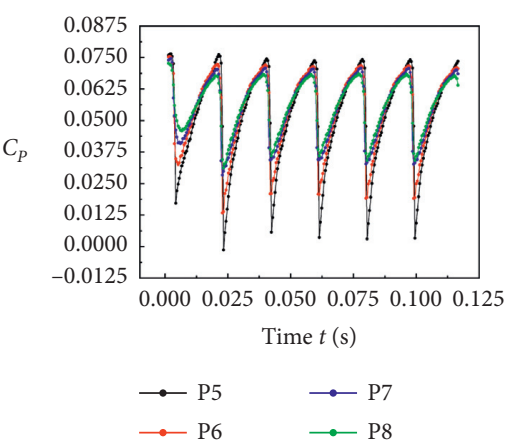

(a)

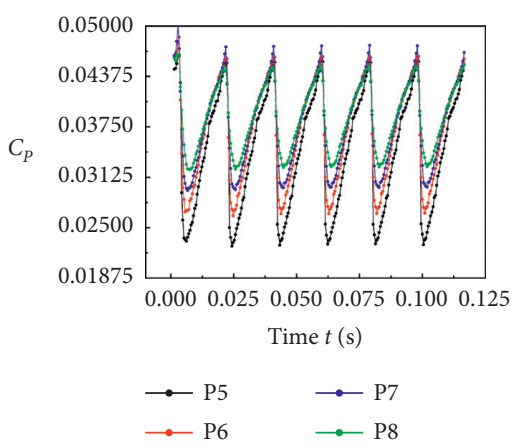

(b)

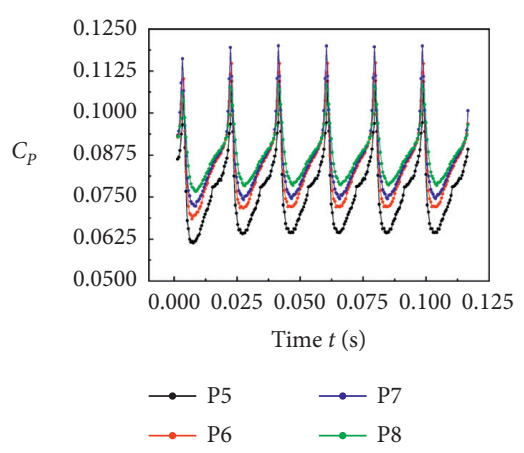

(c)

Figure 24: Time domain distribution of the shaft tubular pump without IGVs under (a) $0.75 Q_{\mathrm{d}}$, (b) $Q_{\mathrm{d}}$, and (c) $1.25 Q_{\mathrm{d}}$.

the blade is greatly reduced. The maximum pressure fluctuation intensity of the vane pressure side (ignoring the blade head and tail area) and suction side of the vertical well tubular pump without IGVs is approximately 1.3 times and 1.1 times that of the IGVs scheme.

To analyze the effect of the IGVs on the pressure pulsation of the impeller inlet of the pump device, time domain diagrams at four monitoring points along the radial distribution of the impeller inlet section are taken out for research, as shown in Figures 24 and 25. The time domain diagrams of the two pumps are similar in the change trend under the same flow conditions. In a rotation period, the time domain graph has three peaks and troughs. Along the direction from the hub to the shroud, the pressure pulsation amplitude at the inlet of the impeller of the two pumps gradually increases. When the flow rate gradually increases, the pressure pulsation amplitude of the impeller inlet of the two pumps first decreases and then increases. The reason is that the pressure difference between the inlet and outlet of the impeller is large under the small-flow condition, and the 


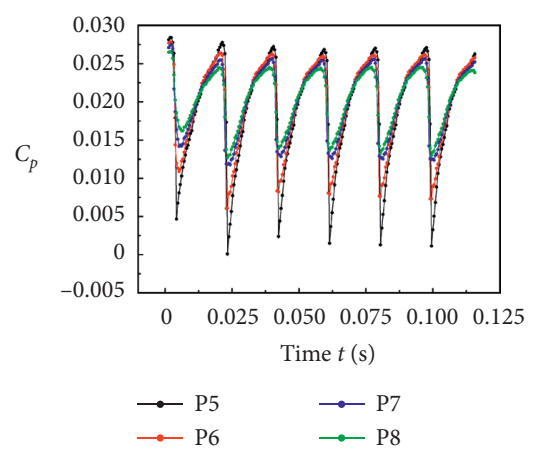

(a)

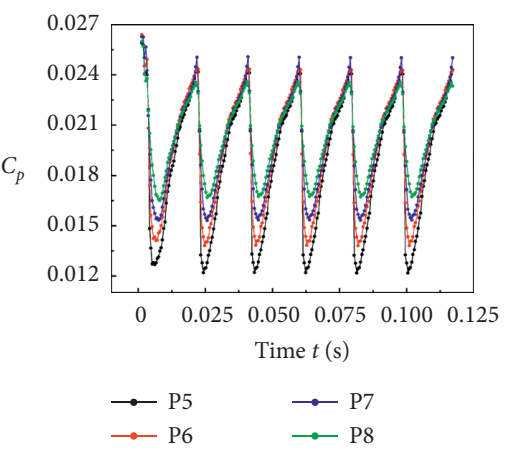

(b)

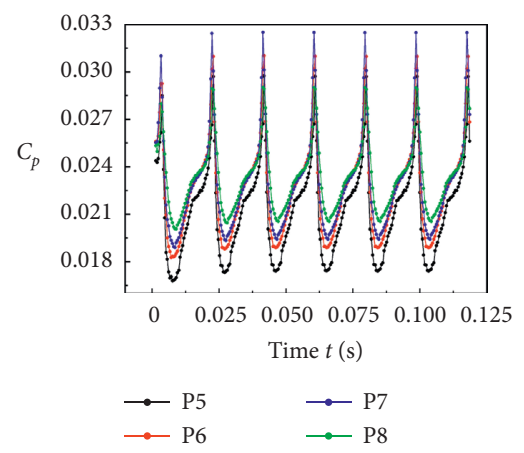

(c)

FIgURE 25: Time domain distribution of the shaft tubular pump with 5 IGVs under (a) $0.75 Q_{d}$, (b) $Q_{d}$, and (c) $1.25 Q_{d}$.

tip clearance backflow is serious. Therefore, the impact of backflow and incoming flow results in a turbulent flow pattern at the inlet. It can also be found that the pressure pulsation amplitude of the impeller inlet of the pump device without IGVs is smaller than that of the IGV scheme under all working conditions. In the IGV scheme, the pressure pulsation amplitude of the impeller inlet $\mathrm{P} 5$ of the pump at $0.75 Q_{\mathrm{d}}, Q_{\mathrm{d}}$, and $1.25 Q_{\mathrm{d}}$ is approximately $1 / 3 /, 3 / 5$, and $3 / 10$ of the scheme without IGVs.

\section{Conclusion}

First, the influence of the number of IGVs on the performance and pressure fluctuation of the shaft tubular pump was experimentally studied. Then, numerical simulations of the shaft tubular pump with and without IGVs were performed by CFD. The influence of the IGVs on the hydraulic performance and pressure fluctuation characteristics of the shaft tubular pump was studied. Finally, tests on the external characteristics and pressure fluctuation were performed to prove the accuracy of the calculation data. Based on the analysis of the pressure fluctuation at the impeller inlet, the following conclusions were obtained:

(1) The number of IGVs has a great impact on the performance of the shaft tubular pump. With the increase in the number of IGVs, the pressure pulsation amplitude at the inlet of the impeller decreases first and then increases under small-flow conditions and design conditions but gradually increases under large-flow conditions.

(2) After the IGVs are added to the impeller inlet, the hydraulic losses of the inlet channel and the IGVs section increase. At the same time, the head and efficiency of the pump device are reduced. The IGVs can improve the uniformity of the impeller inlet flow and improve the impeller water inlet condition, but they have a negative impact on the impeller inlet axial speed.

(3) The impeller inlet is the area with the largest pressure pulsation amplitude inside the shaft tubular pump device, followed by the impeller outlet. Since the IGVs and the guide vanes are far from the impeller, the pressure pulsation amplitude is very small. After adding the IGVs, the overall pressure pulsation value of the shaft tubular pump is reduced. In particular, the pressure pulsation at the inlet of the impeller is significantly weakened.

(4) The advantage of the IGVs is that they reduce the pressure pulsation of the pump device and improve the structure and operation stability of the pump device. The disadvantage is that the head and efficiency are slightly reduced. For the drainage pumping station, due to the short running time of the pumping station, its economic operation is secondary to safety and stability. In summary, the advantages of installing IGVs at the inlet of the shaft tubular pump are more favorable than the disadvantages.

(5) The results for the numerical simulations and model tests of the shaft tubular pumps are in good agreement. This shows that it is accurate and feasible to study the hydraulic performance and pressure fluctuation characteristics of shaft tubular pumps by numerical simulation methods.

\section{Abbreviations}

$\begin{array}{ll}n: & \text { Impeller revolution, r/min } \\ d_{r}: & \text { Hub ratio } \\ d: & \text { Tip clearance } \\ e_{S}: & \text { System error } \\ e_{R}: & \text { Measurement error } \\ C_{p}: & \text { Pressure pulsation coefficient } \\ C_{p}^{*}: & \text { Pressure pulsation intensity } \\ D: & \text { Impeller diameter, mm } \\ P: & \text { Instantaneous pressure, Pa } \\ Q: & \text { Flow, L/s } \\ \rho: & \text { Liquid density, kg/m } 3 \\ \eta: & \text { Efficiency, \% } \\ P: & \text { Average pressure, Pa } \\ u: & \text { Circumferential velocity, } \mathrm{rad} / \mathrm{s} \\ Q_{d}: & \text { Design flow, L/s } \\ H: & \text { Head, m } \\ D_{1}: & \text { Hub diameter, mm }\end{array}$


$u_{i}, u_{j}$ : Time-averaged velocity component

$\alpha_{k}, \alpha_{\varepsilon}$ : The coefficients of $k$ and $\varepsilon$

$U^{\prime}$ : Velocity component

$\mu_{t}: \quad$ Turbulent viscosity

$x_{i}, x_{j}$ : Coordinate component

$\bar{B}: \quad$ Sum of additional forces

$\mu_{e f f}$ : Effective turbulent flow viscosity coefficient

$P^{\prime}: \quad$ Static pressure, $\mathrm{Pa}$

$V_{\mathrm{u}}$ : $\quad$ Flow uniformity, \%

$V_{a i}: \quad$ Axial velocity of the $i$ th grid node, $\mathrm{m} / \mathrm{s}$

$\theta$ : Velocity-weighted average angle, ${ }^{\circ}$

$V_{a}: \quad$ Average axial velocity, $\mathrm{m} / \mathrm{s}$

$\triangle H: \quad$ Average static pressure, $\mathrm{Pa}$

$V_{t i}$ : Lateral velocity of the $i$ th grid node, $\mathrm{m} / \mathrm{s}$

$Z$ : $\quad$ Distance from the section to the central section of the impeller, $\mathrm{mm}$

$m$ : Number of grids of the impeller inlet section.

\section{Data Availability}

The data can be obtained upon request by contacting the corresponding author.

\section{Conflicts of Interest}

The authors declare that this article does not have any potential conflicts of interest.

\section{Authors' Contributions}

Sorting and analysis of test and calculation data were carried out by Haifeng Jiao. Analysis and writing of the paper were performed by Songshan Chen. Chong Sun reviewed and edited the article.

\section{Acknowledgments}

This work was supported by the National Natural Science Foundation of China (51076136), National Science and Technology Support Plan (2006BAB04A03), Natural Science Foundation of Jiangsu Province (BK2008217), Key Programs of Water Conservancy Science and Technology in Jiangsu Province (Grant no. 2009053), and the Priority Academic Program Development of Jiangsu Higher Education Institutions (Grant no. PAPD).

\section{References}

[1] F. Meng, J. Pei, Y. J. Li, and S. Yuan, "Effect of guide vane position on hydraulic performance of two-direction tubular pump device," Journal of Agricultural Machinery, vol. 48, no. 2, pp. 135-140, 2017.

[2] R. S. Xie, F. P. Tang, C. Liu, and F. Yang, "Comparison of hydraulic performance between vertical shaft and shaft extension tubular pumping system," Journal of Agricultural Machinery, vol. 32, no. 13, pp. 24-30, 2016.

[3] Y. Fan, C. Liu, and L. W. Zhang, "Pressure pulsations of the blade region in S-shaped shaft-extension tubular pumping system," Mathematical Problems in Engineering, vol. 2014, Article ID 820135, 10 pages, 2014.

[4] L. J. Shi, X. Q. Liu, F. P. Tang, and Y. Yao, "Design optimization and experimental analysis of bidirectional shaft tubular pump device," Journal of Agricultural Machinery, vol. 47, no. 12, pp. 85-91, 2016.

[5] J. Liu, Y. Zheng, D. Q. Zhou, and Y. Mao, "Analysis of basic flow pattern in shaft front-positioned and shaft rear-positioned tubular pump systems," Journal of Agricultural Machinery, vol. 41, no. S1, pp. 32-38, 2010.

[6] F. H. Han, Z. Wang, Y. J. Mao, T. Jiajian, and W. Li, "Experimental and numerical studies on the influence of inlet guide vanes of centrifugal compressor on the flow field characteristics of inlet chamber," Advances in Mechanical Engineering, vol. 12, no. 11, 2020.

[7] Y. Du, S. X. Zheng, K. Chen et al., "Exergy loss characteristics of a recuperated gas turbine and Kalina combined cycle system using different inlet guide vanes regulation approaches," Energy Conversion and Management, vol. 230, Article ID 113805, 2021.

[8] Y. Q. Ye and J. G. Yang, "Analysis on the abrupt vibration caused by angular deviation of inlet guide vane of gas turbine," Journal of Engineering for thermal Energy and Power, vol. 35, no. 7, pp. 43-48, 2020.

[9] B. Dewar, M. Creamer, M. Dotcheva, J. Radulovic, and J. M. Buick, "A 1D mean line model for centrifugal compressors with variable inlet guide vanes," Engineering Reports, vol. 1, no. 1, 2019.

[10] J. Fukutomi and R. Nakamura, "Performance and internal flow of cross-flow fan with inlet guide vane," The Japan Society of Mechanical Engineers, vol. 48, no. 4, 2005.

[11] M. Coppinger and E. Swain, "Performance prediction of an industrial centrifugal compressor inlet guide vane system," Proceedings of the Institution of Mechanical Engineers-Part A: Journal of Power and Energy, vol. 214, no. 2, 2000.

[12] K. Kabalyk, M. Jasek, G. Liskiewicz, and L. Horodko, "Experimental analysis of the influence of outlet network volume and inlet guide vane positioning on surge behavior in a singlestage low-speed centrifugal compressor," Proceedings of the Institution of Mechanical Engineers-Part A: Journal of Power and Energy, vol. 232, no. 4, p. 4, 2018.

[13] V. Barzdaitis, P. Mažeika, M. Vasylius, V. Kartašovas, and T. Artūras, "Investigation of pressure pulsations in centrifugal pump system," Journal of Vibroengineering, vol. 18, no. 3, pp. 1849-1860, 2016.

[14] R. Spence and J. Amaral-Teixeira, "Investigation into pressure pulsations in a centrifugal pump using numerical methods supported by industrial tests," Computers \& Fluids, vol. 37, no. 6, 2007.

[15] R. Barrio, J. Parrondo, and E. Blanco, "Numerical analysis of the unsteady flow in the near-tongue region in a volute-type centrifugal pump for different operating points," Computers \& Fluids, vol. 39, no. 5, 2010. 
[16] Y. C. Wang, L. Tan, B. S. Zhu, C. ShuLiang, and W. Binbin, "Numerical investigation of influence of inlet guide vanes on unsteady flow in a centrifugal pump," Proceedings of the Institution of Mechanical Engineers-Part C: Journal of Mechanical Engineering Science, vol. 229, no. 18, 2015.

[17] M. Liu, L. Tan, and S. L. Cao, "Influence of geometry of inlet guide vanes on pressure fluctuations of a centrifugal pump," Journal of Fluids Engineering, vol. 140, no. 9, 2018.

[18] L. Shi, J. Zhu, Y. Yuan et al., "Numerical simulation and experiment of the effects of blade angle deviation on the hydraulic characteristics and pressure pulsation of an axialflow pump," Shock and Vibration, vol. 2021, pp. 1-14, Article ID 6673002, 2021.

[19] F. Yang, C. Liu, F. P. Tang, W.-Z. Hu, and Y. Jin, "Numerical simulation on hydraulic performance of axial-flow pumping system with adjustable inlet guide vanes," Transactions of the Chinese Society for Agricultural Machinery, vol. 45, no. 5, pp. 51-58, 2014.

[20] L. Xu, D. Ji, W. Shi, B. Xu, W. Lu, and L. Lu, "Influence of inlet angle of guide vane on hydraulic performance of an axial flow pump based on CFD," Shock and Vibration, vol. 2020, pp. 1-16, Article ID 8880789, 2020.

[21] F. Wang, Z. D. Qian, and Z. W. Guo, "Pressure oscillations prediction of axial flow pump with adjustable guide vanes," Transactions of the Chinese Society for Agricultural Machinery, vol. 48, no. 3, pp. 119-123, 2017.

[22] B. Maxime, K. Kan, H. X. Chen et al., "Flow instability transferability characteristics within a reversible pump turbine (RPT) under large guide vane opening (GVO)," Renewable Energy, vol. 179, pp. 285-307, 2021.

[23] J. Jesline, R. Mehrdad, and C. Michel J, "Study of flow characteristics inside francis turbine draft tube with adjustable guide vanes," IOP Conference Series: Earth and Environmental Science, vol. 774, no. 1, 2021.

[24] M. Binama, W. Su, W. H. Cai et al., "Investigation on reversible pump turbine flow structures and associated pressure field characteristics under different guide vane openings," Science China Technological Sciences, vol. 62, no. 11, 2019.

[25] S. Chitrakar, O. G. Dahlhaug, and H. P. Neopane, "Numerical investigation of the effect of leakage flow through erosioninduced clearance gaps of guide vanes on the performance of Francis turbines," Engineering Applications of Computational Fluid Mechanics, vol. 12, no. 1, 2018.

[26] S. Chitrakar, B. S. Thapa, O. G. Dahlhaug, and H. P. Neopane, "Numerical investigation of the flow phenomena around a low specific speed Francis turbine's guide vane cascade," IOP Conference Series: Earth and Environmental Science, vol. 49, no. 6, 2016.

[27] F. Yang, W.-z. Hu, C. Li, C. Liu, and Y. Jin, "Computational study on the performance improvement of axial-flow pump by inlet guide vanes at part loads," Journal of Mechanical Science and Technology, vol. 34, no. 12, pp. 4905-4915, 2020.

[28] Z. L. Zhao, Z. W. Guo, Z. D. Qian, and Q. Cheng, "Performance improvement of an axial-flow pump with inlet guide vanes in the turbine mode," Proceedings of the Institution of Mechanical Engineers-Part A: Journal of Power and Energy, vol. 234, no. 3, 2020.

[29] Y. S. Kim, H.-S. Shim, and K. Kwang-Yong, "Effects of inlet guide vane and blade pitch angles on the performance of a submersible axial-flow pump," Proceedings of the Institution of Mechanical Engineers-Part A: Journal of Power and Energy, vol. 235, no. 4, 2021.

[30] Z. W. Guo, J. Y. Pan, Z. D. Qian, and J. Bin, "Experimental and numerical analysis of the unsteady influence of the inlet guide vanes on cavitation performance of an axial pump," Proceedings of the Institution of Mechanical Engineers-Part C: Journal of Mechanical Engineering Science, vol. 233, no. 11, pp. 3816-3826, 2019.

[31] H. Yang, D. D. Sun, and F. P. Tang, "Research on the performance improvement of axial-flow pump under unstable condition using CFD," Transactions of the Chinese Society for Agricultural Machinery, vol. 43, no. 11, pp. 138-141+151, 2012.

[32] Y. Y. Xing, R. S. Xie, and C. Xiang, "Simulation study on improving saddle zone of axial flow pump device with front baffle," Coal Mine Machinery, vol. 40, no. 01, pp. 52-54, 2019.

[33] J. Dai, Y. J. Li, and S. Q. Yuan, "Effect of inlet guide vane position on hydraulic performance of bulb tubular pump," Journal of Drainage and Irrigation Machinery Engineering, vol. 35, no. 9, pp. 761-766, 2017.

[34] J. Deng, J. L. Lu, Y. R. Xu, F. JianJun, and L. XingQi, "Effect of clocking position of front guide vane on pulsation characteristic of pressure in centrifugal pump," Journal of Northwest Forestry University, vol. 44, no. 1, pp. 217-222, 2016.

[35] X. Song and C. Liu, "Experimental investigation of floorattached vortex effects on the pressure pulsation at the bottom of the axial flow pump sump," Renewable Energy, vol. 145, pp. 2327-2336, 2020.

[36] X. H. Duan, F. P. Tang, W. Y. Duan, W. Zhou, and L. Shi, "Experimental investigation on the correlation of pressure pulsation and vibration of axial flow pump," Advances in Mechanical Engineering, vol. 11, no. 11, 2019.

[37] L. Xu, L. G. Lu, W. Chen, and G. Wang, "Flow pattern analysis on inlet and outlet conduit of shaft tubular pump system of Pizhou pumping station in South-to-North Water Diversion Project," Transactions of the Chinese Society of Agricultural Engineering, vol. 28, no. 6, pp. 50-56, 2012.

[38] R. S. Xie, Z. Wu, and Y. He, "Optimization research on passage of bidirectional shaft tubular pump," Transactions of the Chinese Society for Agricultural Machinery, vol. 46, no. 10, pp. 68-74, 2015.

[39] A. R. Al-Obaidi, "Investigation of the influence of various numbers of impeller blades on internal flow field analysis and the pressure pulsation of an axial pump based on transient flow behavior," Heat Transfer, vol. 49, no. 4, 2020.

[40] L. Shi, W. Zhang, H. Jiao et al., "Numerical simulation and experimental study on the comparison of the hydraulic characteristics of an axial-flow pump and a full tubular pump," Renewable Energy, vol. 153, pp. 1455-1464, 2020.

[41] X. J. Song and C. Liu, "Experimental investigation of pressure pulsation induced by the floor-attached vortex in an axial flow pump," Advances in Mechanical Engineering, vol. 11, no. 3, 2019.

[42] L. Shi, Y. Yuan, H. Jiao et al., "Numerical investigation and experiment on pressure pulsation characteristics in a full tubular pump," Renewable Energy, vol. 163, pp. 987-1000, 2021.

[43] C. L. Xie, F. P. Tang, R. T. Zhang, W. Zhou, W. Zhang, and F. Yang, "Numerical calculation of axial-flow pump's pressure fluctuation and model test analysis," Advances in Mechanical Engineering, vol. 10, no. 4, 2018.

[44] W. P. Zhang, F. P. Tang, L. J. Shi, Q. Hu, and Y. Zhou, "Effects of an inlet vortex on the performance of an axial-flow pump," Energies, vol. 13, no. 11, 2020.

[45] C. Z. Xia, L. Cheng, H. Y. Jiang, and J. Xin, "Hydraulic performance analysis and optimization on flow passage components of diving tubular pumping system," Transactions 
of the Chinese Society for Agricultural Machinery, vol. 34, no. 7, pp. 45-51+301, 2018.

[46] T. Mu, R. Zhang, H. Xu, Y. Zheng, Z. Fei, and J. Li, "Study on improvement of hydraulic performance and internal flow pattern of the axial flow pump by groove flow control technology," Renewable Energy, vol. 160, pp. 756-769, 2020. 